\title{
Optimization of Collective Reduction Operations
}

\author{
Rolf Rabenseifner \\ High-Performance Computing-Center (HLRS), University of Stuttgart \\ Allmandring 30, D-70550 Stuttgart, Germany \\ rabenseifner@hlrs.de, \\ ww.hlrs.de/people/rabenseifner/
}

\begin{abstract}
A 5-year-profiling in production mode at the University of Stuttgart has shown that more than $40 \%$ of the execution time of Message Passing Interface (MPI) routines is spent in the collective communication routines MPI_Allreduce and MPI_Reduce. Although MPI implementations are now available for about 10 years and all vendors are committed to this Message Passing Interface standard, the vendors' and publicly available reduction algorithms could be accelerated with new algorithms by a factor between 3 (IBM, sum) and 100 (Cray T3E, maxloc) for long vectors. This paper presents five algorithms optimized for different choices of vector size and number of processes. The focus is on bandwidth dominated protocols for power-of-two and non-power-of-two number of processes, optimizing the load balance in communication and computation.
\end{abstract}

Keywords: Message Passing, MPI, Collective Operations, Reduction.

\section{Introduction and Related Work}

MPI_Reduce combines the elements provided in the input vector (buffer) of each process using an operation (e.g. sum, maximum), and returns the combined values in the output buffer of a chosen process named root. MPI_Allreduce is the same as MPI_Reduce, except that the result appears in the receive buffer of all processes. MPI_Allreduce is one of the most important MPI routines and most vendors are using algorithms that can be improved by a factor of more than 2 for long vectors. Most current implementations are optimized only for short vectors. A 5-year-profiling [11] of most MPI based applications (in production mode) of all users of the Cray T3E 900 at our university has shown, that $8.54 \%$ of the execution time is spent in MPI routines. $37.0 \%$ of the MPI time is spent in MPI_Allreduce and 3.7\% in MPI_Reduce. The 5-year-profiling has also shown, that $25 \%$ of all execution time was spent with a non-power-of-two number of processes. Therefore, a second focus is the optimization for non-power-of-two numbers of processes.

Early work on collective communication implements the reduction operation as an inverse broadcast and do not try to optimize the protocols based on different buffer sizes [1]. Other work already handle allreduce as a combination of basic 
routines, e.g., 2] already proposed the combine-to-all (allreduce) as a combination of distributed combine (reduce_scatter) and collect (allgather). Collective algorithms for wide-area cluster are developed in 578, further protocol tuning can be found in 34912, and automatic tuning in [13. The main focus of the work presented in this paper is to optimize the algorithms for different numbers of processes (non-power-of-two and power-of-two) and for different buffer sizes by using special reduce_scatter protocols without the performance penalties on normal rank-ordered scattering. The allgather protocol is chosen according the the characteristics of the reduce_scatter part to achieve an optimal bandwidth for any number of processes and buffer size.

\section{Allreduce and Reduce Algorithms}

\subsection{Cost Model}

To compare the algorithms, theoretical cost estimation and benchmark results are used. The cost estimation is based on the same flat model used by R. Thakur and B. Gropp in [12]. Each process has an input vector with $n$ bytes, $p$ is the number of MPI processes, $\gamma$ the computation cost per vector byte executing one operation with two operands locally on any process. The total reduction effort is $(p-1) n \gamma$. The total computation time with optimal load balance on $p$ processes is therefore $\frac{p-1}{p} n \gamma$, i.e., less than $n \gamma$, which is independent of the number of processes! The communication time is modeled as $\alpha+n \beta$, where $\alpha$ is the latency (or startup time) per message, and $\beta$ is the transfer time per byte, and $n$ the message size in bytes. It is assumed further that all processes can send and receive one message at the same time with this cost model. In reality, most networks are faster, if the processes communicate in parallel, but pairwise only in one direction (uni-directional between two processes), e.g., in the classical binary tree algorithms. Therefore $\alpha_{u n i}+n \beta_{u n i}$ is modeling the uni-directional communication, and $\alpha+n \beta$ is used with the bi-directional communication. The ratios are abbreviated with $f_{\alpha}=\alpha_{u n i} / \alpha$ and $f_{\beta}=\beta_{u n i} / \beta$. These factors are normally in the range 0.5 (simplex network) to 1.0 (full duplex network).

\subsection{Principles}

A classical implementation of MPI_Allreduce is the combination of MPI_Reduce (to a root process) followed by MPI_Bcast sending the result from root to all processes. This implies a bottle-neck on the root process. Also classical is the binary tree implementation of MPI_Reduce, which is a good algorithm for short vectors, but that causes a heavy load imbalance because in each step the number of active processes is halved. The optimized algorithms are based on a few principles:

Recursive vector halving: For long-vector reduction, the vector can be split into two parts and one half is reduced by the process itself and the other half is sent to a neighbor process for reduction. In the next step, again the buffers are halved, and so on. 
Recursive vector doubling: To return the total result in the result vector, the split result vectors must be combined recursively. MPI_Allreduce can be implemented as a reduce-scatter (using recursive vector halving) followed by an allgather (using recursive vector doubling).

Recursive distance doubling: In step 1, each process transfers data at distance 1 (process $\mathrm{P} 0$ with $\mathrm{P} 1, \mathrm{P} 2-\mathrm{P} 3, \mathrm{P} 4-\mathrm{P} 5, \ldots$ ); in step 2, the distance is doubled, i.e., $\mathrm{P} 0-\mathrm{P} 2$ and $\mathrm{P} 1-\mathrm{P} 3, \mathrm{P} 4-\mathrm{P} 6$ and $\mathrm{P} 5-\mathrm{P} 7$; and so on until distance $\frac{p}{2}$. Recursive distance halving: Same procedure, but starting with distance $p / 2$, i.e., $\mathrm{P} 0-\mathrm{P} \frac{p}{2}, \mathrm{P} 1-\mathrm{P}\left(\frac{p}{2}+1\right), \ldots$, and ending with distance 1 , i.e., $\mathrm{P} 0-\mathrm{P} 1, \ldots$.

Recursive vector and distance doubling and halving can be combined for different purposes, but always additional overhead causes load imbalance if the number of processes is not a power of two. Two principles can reduce the overhead in this case.

Binary blocks: The number of processes can be expressed as a sum of power-oftwo values, i.e., all processes are located in subsets with power-of-two processes. Each subset is used to execute parts of the reduction protocol in a block. Overhead occurs in the combining of the blocks in some step of the protocol.

Ring algorithms: A reduce_scatter can be implemented by $p-1$ ring exchange steps with increasing strides. Each process computes all reduction operations for its own chunk of the result vector. In step $i(i=1 . . p-1)$ each process sends the input vector chunk needed by rank $+i$ to that process and receives from $r a n k-i$ the data needed to reduce its own chunk. The allreduce can be completed by an allgather that is also implemented with ring exchange steps, but with constant stride 1. Each process sends its chunk of the result vector around the ring to the right $(\operatorname{rank}+1)$ until its left neighbor $((\operatorname{ran} k+p-1) \bmod p)$ has received it after $p-1$ steps. The following sections describe the algorithms in detail.

\subsection{Binary Tree}

Reduce: The classical binary tree always exchanges full vectors, uses recursive distance doubling, but with incomplete protocol, because in each step, half of the processes finish their work. It takes $\lceil\lg p\rceil$ steps and the time taken by this algorithm is $\left.T_{\text {red,tree }}=\lceil\lg p\rceil\left(\alpha_{u n i}+n \beta_{\text {uni }}+n \gamma\right)\right)$.

For short vectors, this algorithm is optimal (compared to the following algorithms) due to its smallest latency term $\lceil\lg p\rceil \alpha_{\text {uni }}$.

Allreduce: The reduce algorithm is followed by a binary tree based broadcast. The total execution time is $\left.T_{\text {all,tree }}=\lceil\lg p\rceil\left(2 \alpha_{u n i}+2 n \beta_{u n i}+n \gamma\right)\right)$.

\subsection{Recursive Doubling}

Allreduce: This algorithm is an optimization especially for short vectors. In each step of the recursive distance doubling, both processes in a pair exchange the input vector (in step 1) or its intermediate result vector (in steps $2 \ldots\lceil\lg p\rceil$ ) with its partner process and both processes are computing the same reduction redundantly. After $\lceil\lg p\rceil$ steps, the identical result vector is available in all processes. It needs $\left.T_{a l l, r . d .}=\lceil\lg p\rceil(\alpha+n \beta+n \gamma)\right)+\left(\right.$ if non-power-of-two $\left.\alpha_{\text {uni }}+n \beta_{\text {uni }}\right)$ This algorithm is in most cases optimal for short vectors. 


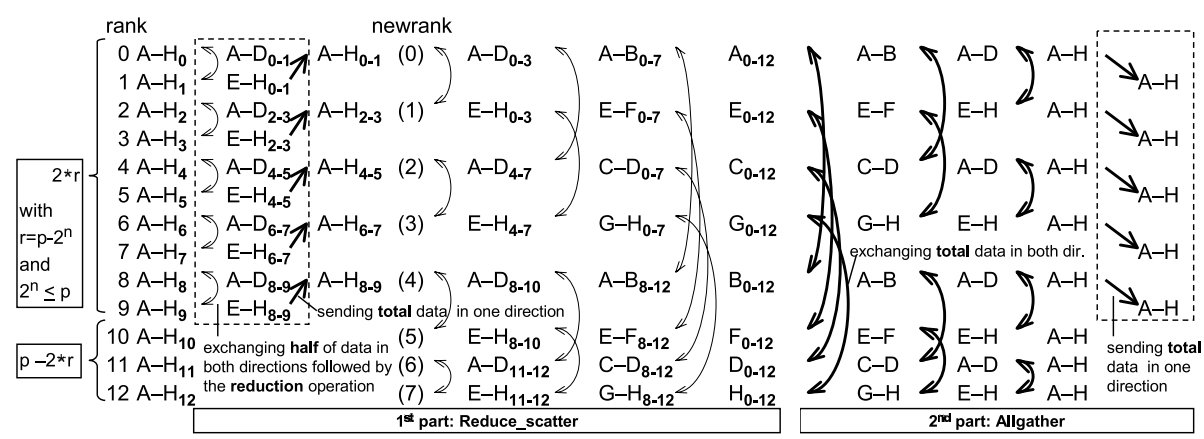

Fig. 1. Recursive Halving and Doubling. The figure shows the intermediate results after each buffer exchange (followed by a reduction operation in the $1^{\text {st }}$ part). The dotted frames show the overhead caused by a non-power-of-two number of processes

\subsection{Recursive Halving and Doubling}

This algorithm is a combination of a reduce_scatter implemented with recursive vector halving and distance doubling 1 followed by a allgather implemented by a recursive vector doubling combined with recursive distance halving (for allreduce), or followed by gather implemented with a binary tree (for reduce).

In a first step, the number of processes $p$ is reduced to a power-of-two value: $p^{\prime}=2^{\lfloor\lg p\rfloor} \cdot r=p-p^{\prime}$ is the number of processes that must be removed in this first step. The first $2 r$ processes send pairwise from each even rank to the odd $($ rank +1$)$ the second half of the input vector and from each odd rank to the even $($ rank -1$)$ the first half of the input vector. All $2 r$ processes compute the reduction on their half.

Fig. 1] shows the protocol with an example on 13 processes. The input vectors and all reduction results will be divided into $p^{\prime}$ parts $(\mathrm{A}, \mathrm{B}, \ldots, \mathrm{H})$ by this algorithm, and therefore it is denoted with $\mathrm{A}-\mathrm{H}_{\text {rank }}$. After the first reduction, process $\mathrm{P} 0$ has computed $\mathrm{A}-\mathrm{D}_{0-1}$, denoting the reduction result of the first half of the vector (A-D) from the processes $0-1$. P1 has computed $\mathrm{E}-\mathrm{H}_{0-1}, \mathrm{P} 2$ $\mathrm{A}-\mathrm{D}_{2-3}, \ldots$. The first step is finished by sending those results from each odd process $(1 \ldots 2 r-1)$ to rank -1 into the second part of the buffer.

Now, the first $r$ even processes and the $p-2 r$ last processes are renumbered from 0 to $p^{\prime}-1$. This first step needs $\left(1+f_{\alpha}\right) \alpha+\frac{1+f_{\text {beta }}}{2} n \beta+\frac{1}{2} n \gamma$ and is not necessary, if the number of processes $p$ was already a power-of-two.

Now, we start with the first step of recursive vector halving and distance doubling, i.e., the even / odd ranked processes are sending the second / first half

\footnotetext{
${ }^{1}$ A distance doubling (starting with distance 1) is used in contrary to the reduce_scatter algorithm in [12] that must use a distance halving (i.e., starting with distance $\frac{\# \text { processes }}{2}$ ) to guarantee a rank-ordered scatter. In our algorithm, any order of the scattered data is allowed, and therefore, the longest vectors can be exchanged with the nearest neighbor, which is an additional advantage on systems with a hierarchical network structure.
} 
of their buffer to $r a n k^{\prime}+1 / r^{\prime} a n k^{\prime}-1$. Then the reduction is computed between the local buffer and the received buffer. This step costs $\alpha+\frac{1}{2}(n \beta+n \gamma)$.

In the next $\lg p^{\prime}-1$ steps, the buffers are recursively halved and the distance doubled. Now, each of the $p^{\prime}$ processes has $\frac{1}{p^{\prime}}$ of the total reduction result vector, i.e., the reduce_scatter has scattered the result vector to the $p^{\prime}$ processes. All recursive steps cost $\lg p^{\prime} \alpha+\left(1-\frac{1}{p^{\prime}}\right)(n \beta+n \gamma)$. The second part implements an allgather or gather to complete the allreduce or reduce operation.

Allreduce: Now, the contrary protocol is needed: Recursive vector doubling and distance halving, i.e., in the first step the process pairs exchange $\frac{1}{p^{\prime}}$ of the buffer to achieve $\frac{2}{p^{\prime}}$ of the result vector, and in the next step $\frac{2}{p^{\prime}}$ is exchanged to get $\frac{4}{p^{\prime}}$, and so on. A-B, A-D ... in Fig. प denote the already stored portion of the result vector. After each communication exchange step, the result buffer is doubled and after $\lg p^{\prime}$ steps, the $p^{\prime}$ processes have received the total reduction result. This allgather part costs $\lg p^{\prime} \alpha+\left(1-\frac{1}{p^{\prime}}\right)(n \beta)$.

If the number of processes is non-number-of-two, then the total result vector must be sent to the $r$ removed processes. This causes the additional overhead $\alpha+n \beta$. The total implementation needs

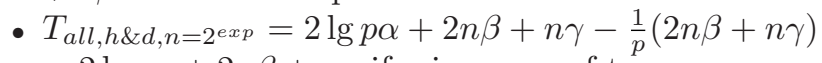
$\simeq 2 \lg p \alpha+2 n \beta+n \gamma$ if $p$ is power-of-two,

- $T_{\text {all }, h \& d, n \neq 2^{\text {exp }}}=\left(2 \lg p^{\prime}+2+f_{\alpha}\right) \alpha+\left(3+\frac{1+f_{\text {beta }}}{2}\right) n \beta+\frac{3}{2} n \gamma-\frac{1}{p^{\prime}}(2 n \beta+n \gamma)$ $\simeq(3+2\lfloor\lg p\rfloor) \alpha+4 n \beta+\frac{3}{2} n \gamma$ if $p$ is non-power-of-two (with $\left.p^{\prime}=2^{\lfloor\lg p\rfloor}\right)$.

This protocol is good for long vectors and power-of-two processes. For nonpower-of-two processes, the transfer overhead is doubled and the computation overhead is enlarged by $\frac{3}{2}$. The binary blocks protocol (see below) can reduce this overhead in many cases.

Reduce: The same protocol is used, but the pairwise exchange with sendrecv is substituted by single message passing. In the first step, each process with the bit with the value $p^{\prime} / 2$ in its new rank identical to that bit in root rank must receive a result buffer segment and the other processes must send their segment. In the next step only the receiving processes continue and the bit is shifted 1 position right (i.e., $\left.p^{\prime} / 4\right)$. And so on. The time needed for this gather operation is $\lg p^{\prime} \alpha_{u n i}+\left(1-\frac{1}{p^{\prime}}\right) n \beta_{u n i}$.

In the case that the original root process is one of the removed processes, then the role of this process and its partner in the first step are exchanged after the first reduction in the reduce_scatter protocol. This causes no additional overhead. The total implementation needs

- $T_{r e d, h \& d, n=2^{\exp }}=\lg p\left(1+f_{\alpha}\right) \alpha+\left(1+f_{\beta}\right) n \beta+n \gamma-\frac{1}{p}\left(n\left(\beta+\beta_{\text {uni }}\right)+n \gamma\right)$ $\simeq 2 \lg p \alpha+2 n \beta+n \gamma$ if $p$ is power-of-two,

- $T_{\text {red,h\&d,nf2exp }}=\lg p^{\prime}\left(1+f_{\alpha}\right) \alpha+\left(1+f_{\alpha}\right) \alpha+\left(1+\frac{1+f_{\text {beta }}}{2}+f_{\beta}\right) n \beta+\frac{3}{2} n \gamma-$ $\frac{1}{p^{\prime}}\left(\left(1+f_{\beta}\right) n \beta+n \gamma\right)$

$\simeq(2+2\lfloor\lg p\rfloor) \alpha+3 n \beta+\frac{3}{2} n \gamma$ if $p$ is non-power-of-two (with $\left.p^{\prime}=2^{\lfloor\lg p\rfloor}\right)$. 


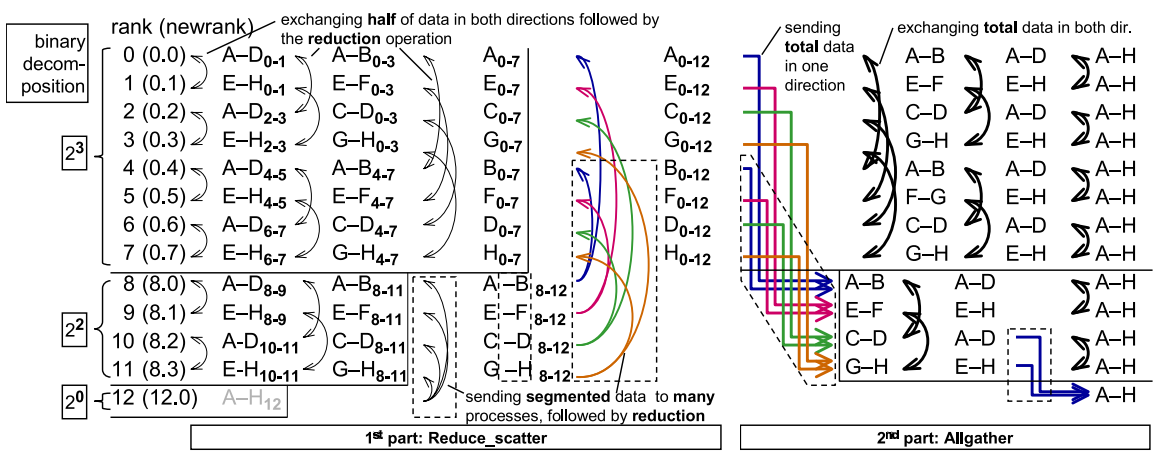

Fig. 2. Binary Blocks

\subsection{Binary Blocks}

Further optimization for non-power-of-two number of processes can be achieved with the algorithm shown in Fig.2.

Here, the maximum difference between the ratio of the number of proccesses of two successive blocks, especially in the low range of exponents, determines the imbalance.

Allreduce: The $2^{\text {nd }}$ part is an allgather implemented with buffer doubling and distance halving in each block as in the algorithm in the previous section. The input must be provided in the processes of the smaller blocks always with pairs of messages from processes of the next larger block.

Reduce: If the root is outside of the largest block, then the intermediate result segment of rank 0 is sent to root and root plays the role of rank 0 . A binary tree is used to gather the result segments into the root process.

For power-of-two number of processes, the binary block algorithms are identical to the halving and doubling algorithm in the previous section.

\section{$2.7 \quad$ Ring}

While the algorithms in the last two sections are optimal for power-of-two process numbers and long vectors, for medium non-power-of-two number of processes and long vectors there exist another good algorithm. It uses the pairwise exchange algorithm for reduce_scatter and ring algorithm for allgather (for allreduce), as described in [12], and for reduce, all processes send their result segment directly to root. Both algorithms are good in bandwidth usage for nonpower-of-two number of processes, but the latency scales with the number of processes. Therefore this algorithm can be used only for a small number of processes. Independent of whether $p$ is power-of-two or not, the total implementation needs $T_{\text {all,ring }}=2(p-1) \alpha+2 n \beta+n \gamma-\frac{1}{p}(2 n \beta+n \gamma)$ for allreduce, and $T_{\text {red,ring }}=(p-1)\left(\alpha+\alpha_{u n i}\right)+n\left(\beta+\beta_{u n i}\right)+n \gamma-\frac{1}{p}\left(n\left(\beta+\beta_{u n i}\right)+n \gamma\right)$ for reduce. 


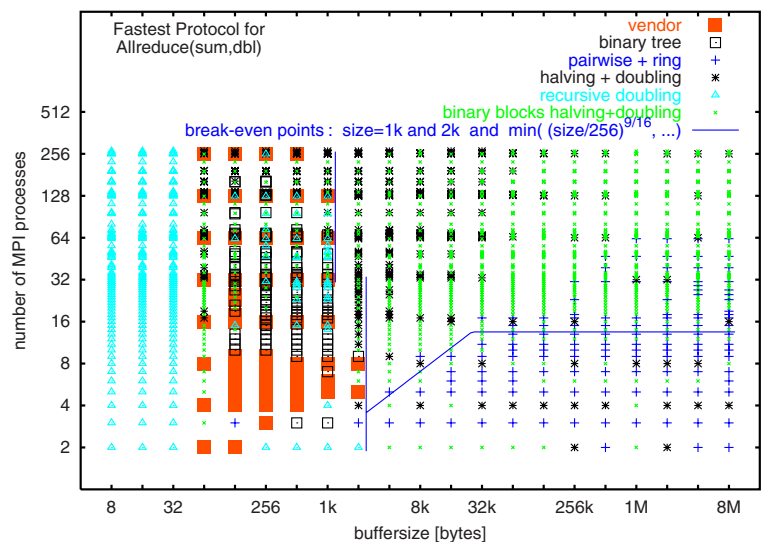

Fig. 3. The fastest protocol for Allreduce(double, sum) on a Cray T3E 900.

\section{Choosing the Fastest Algorithm}

Based on the number of processes and the vector (input buffer) length, the reduction routine must decide which algorithm should be used. Fig. 3] shows the fastest protocol on a Cray T3E 900 with 540 PEs. For buffer sizes less than or equal to 32 byte, recursive doubling is the best, for buffer sizes less than or equal to $1 \mathrm{~KB}$, mainly vendor's algorithm (for power-of-two) and binary tree (for nonpower-of-two) are the best but there is not a big difference to recursive doubling. For longer buffer sizes, the ring is good for some buffer sizes and some \#processes less than 32 PEs. A detailed decision is done for each \#processes value, e.g., for 15 processes, ring is used if length $\geq 64 \mathrm{~KB}$. In general, on a Cray T3E 900, the binary block algorithm is faster if $\delta_{\text {expo,max }}<\lg \left(\frac{\text { vector size }}{1 \text { Byte }}\right) / 2.0-2.5$ and vector size $\geq 16 \mathrm{~KB}$ and more than 32 processes are used. In a few cases, e.g., 33 PEs and less then $32 \mathrm{~KB}$, halving 6 doubling is the fastest algorithm.

Fig.[4 shows that with the pure MPI programming model (i.e., 1 MPI process per CPU) on the IBM SP, the benefit is about $1.5 \mathrm{x}$ for buffer sizes $8-64 \mathrm{~KB}$, and $2 \mathrm{x}-5 \mathrm{x}$ for larger buffers. With the hybrid programming model (1 MPI process per SMP node), only for buffer sizes $4-128 \mathrm{~KB}$ and more than 4 nodes, the benefit is about $1.5 \mathrm{x}-3 \mathrm{x}$.

\section{Conclusions and Future Work}

Although principal work on optimizing collective routines is quite old [2], there is a lack of fast implementations for allreduce and reduce in MPI libraries for a wide range of number of processes and buffer sizes. Based on the author's algorithm from 1997 [10, an efficient algorithm for power-of-two and non-powerof-two number of processes is presented in this paper. Medium non-power-oftwo number of processes could be additionally optimized with a special ring algorithm. The halving\&doubling is already included into MPICH-2 and it is 

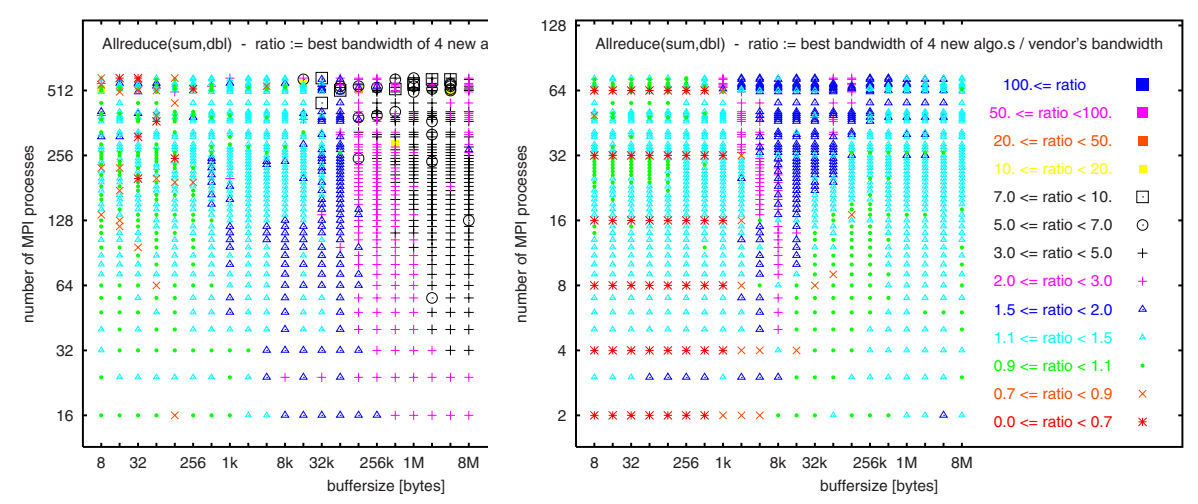

Fig. 4. Ratio of bandwidth of the fastest protocol (without recursive doubling) on a IBM SP at SDSC and 1 MPI process per CPU (left) and per SMP node (right)

planned to include the other bandwidth-optimized algorithms [10]12]. Future work will further optimize latency and bandwidth for any number of processes by combining the principles used in Sect.2.3 2.7 into one algorithm and selecting on each recursion level instead of selecting one of those algorithms for all levels.

Acknowledgments. The author would like to acknowledge his colleagues and all the people that supported this project with suggestions and helpful discussions. He would especially like to thank Rajeev Thakur and Jesper Larsson Träff for the helpful discussion on optimized reduction algorithm and Gerhard Wellein, Thomas Ludwig, Ana Kovatcheva, Rajeev Thakur for their benchmarking support.

\section{References}

1. V. Bala, J. Bruck, R. Cypher, P. Elustondo, A. Ho, C.-T. Ho, S. Kipnis and M. Snir, CCL: A portable and tunable collective communication library for scalable parallel computers, in IEEE Transactions on Parallel and Distributed Systems, Vol. 6, No. 2, Feb. 1995, pp 154-164.

2. M. Barnett, S. Gupta, D. Payne, L. Shuler, R. van de Gejin, and J. Watts, Interprocessor collective communication library (InterCom), in Proceedings of Supercomputing '94, Nov. 1994.

3. Edward K. Blum, Xin Wang, and Patrick Leung, Architectures and message-passing algorithms for cluster computing: Design and performance, in Parallel Computing 26 (2000) 313-332.

4. J. Bruck, C.-T. Ho, S. Kipnis, E. Upfal, and D. Weathersby, Efficient algorithms for all-to-all communications in multiport message-passing systems, in IEEE Transactions on Parallel and Distributed Systems, Vol. 8, No. 11, Nov. 1997, pp 1143-1156.

5. E. Gabriel, M. Resch, and R. Rühle, Implementing MPI with optimized algorithms for metacomputing, in Proceedings of the MPIDC'99, Atlanta, USA, March 1999, pp 31-41. 
6. Message Passing Interface Forum. MPI: A Message-Passing Interface Standard, Rel. 1.1, June 1995, www.mpi-forum.org.

7. N. Karonis, B. de Supinski, I. Foster, W. Gropp, E. Lusk, and J. Bresnahan, Exploiting hierarchy in parallel computer networks to optimize collective operation performance, in Proceedings of the 14th International Parallel and Distributed Processing Symposium (IPDPS '00), 2000, pp 377-384.

8. Thilo Kielmann, Rutger F. H. Hofman, Henri E. Bal, Aske Plaat, Raoul A. F. Bhoedjang, MPI's reduction operations in clustered wide area systems, in Proceedings of the Message Passing Interface Developer's and User's Conference 1999 (MPIDC'99), Atlanta, USA, March 1999, pp 43-52.

9. Man D. Knies, F. Ray Barriuso, William J. Harrod, George B. Adams III, SLICC: A low latency interface for collective communications, in Proceedings of the 1994 conference on Supercomputing, Washington, D.C., Nov. 14-18, 1994, pp 89-96.

10. Rolf Rabenseifner, A new optimized MPI reduce and allreduce algorithm, Nov. 1997. http://www.hlrs.de/mpi/myreduce.html

11. Rolf Rabenseifner, Automatic MPI counter profiling of all users: First results on a CRAY T3E 900-512, Proceedings of the Message Passing Interface Developer's and User's Conference 1999 (MPIDC'99), Atlanta, USA, March 1999, pp 77-85. http://www.hlrs.de/people/rabenseifner/publ/publications.html

12. Rajeev Thakur and William D. Gropp, Improving the performance of collective operations in MPICH, in Recent Advances in Parallel Virtual Machine and Message Passing Interface, proceedings of the 10th European PVM/MPI Users' Group Meeting, LNCS 2840, J. Dongarra, D. Laforenza, S. Orlando (Eds.), 2003, 257-267.

13. Sathish S. Vadhiyar, Graham E. Fagg, and Jack Dongarra, Automatically tuned collective communications, in Proceedings of SC2000, Nov. 2000.

An extended version of this paper can be found on the author's home/publication page. 